\title{
Silicon-based plasmonic photodetector in the near- infrared region with a Schottky barrier properly controlled by nanoalloys
}

Shinya Okamoto ( $\square$ okamoto.shinya001@jp.panasonic.com )

Panasonic Corporation https://orcid.org/0000-0002-4572-2459

Kohei Kusada

Kyoto University https://orcid.org/0000-0002-9679-6749

\section{Yuki Nomura}

Panasonic Corporation https://orcid.org/0000-0002-6091-5902

\section{Eiji Takeda}

Panasonic Corporation https://orcid.org/0000-0002-3328-6610

\section{Yasuhisa Inada}

Panasonic Corporation https://orcid.org/0000-0002-8025-3168

\section{Kazuya Hisada}

Panasonic Corporation

\section{Satoshi Anada}

Japan Fine Ceramics Centre

\section{Kazuo Yamamoto}

Japan Fine Ceramics Center https://orcid.org/0000-0001-7651-943X

\section{Taku Hirasawa}

Panasonic Corporation

Hiroshi Kitagawa

Kyoto University https://orcid.org/0000-0001-6955-3015

\section{Article}

Keywords:

Posted Date: February 2nd, 2022

DOI: https://doi.org/10.21203/rs.3.rs-1231588/v1

License: (1) (1) This work is licensed under a Creative Commons Attribution 4.0 International License.

Read Full License 


\section{Abstract}

Plasmonic Schottky devices have attracted considerable attention for use in practical applications based on photoelectric conversion because they enable to harvest light below the bandgap of semiconductors. In particular, silicon (Si)-based plasmonic Schottky devices have great potential for useful photodetection in the near-infrared region. However, the internal quantum efficiency (IQE) values of previously reported devices have been low because the Schottky barrier is excessively high. Here, we are the first to develop AuAg nanoalloy-n-type Si plasmonic Schottky devices by cathodic arc plasma deposition. These plasmonic nanostructures can be fabricated in only $\sim 1 \mathrm{~min}$. The fabricated AuAg nanoparticles-film structure enables proper control of the Schottky barrier height and increases the area of the Schottky interface for electron transfer. As a result, the considerably enhanced IQE of our device at $1310 \mathrm{~nm}$ (1550 $\mathrm{nm}$ ) is 4.6 (6.5) times higher than those in previous reports, and these responsivities are a record high.

\section{Background}

Plasmon-induced hot carrier generation in plasmonic Schottky devices has been a promising way to harvest light with lower energy than the bandgap of semiconductors, while interband absorption in semiconductors is restricted by their bandgap. Hot electron-hole pairs are created by Landau damping in plasmonic nanostructures when resonant light is irradiated, and some of the hot electrons are injected into the conduction band of the semiconductor over the Schottky barrier formed between the metal and semiconductor ${ }^{1,2}$. Recently, several types of plasmonic Schottky devices with different plasmonic nanostructures, such as nanoparticles $(\mathrm{NPs})^{3-7}$, nanorods ${ }^{8-10}$, chiral metamaterial ${ }^{11}$, periodic gratings ${ }^{12,13}$, and thin metal coated-nanohole ${ }^{14}$ and -trench structures ${ }^{15-17}$ combined with semiconductors, have been demonstrated for a broad range of application fields, such as photodetection, nanoimaging, photovoltaics and photochemistry.

The use of near-infrared (NIR) light has attracted considerable attention as a key to significantly improve conventional applications in the field of imaging, surveillance, and long-distance sensing using eye-safe lasers. Silicon ( $\mathrm{Si}$ ) photodetectors are widely employed in the visible region because complementary metal-oxide-semiconductor (CMOS) technology for the mass production of practical devices is well established; however, it cannot be used for the detection of NIR light below its bandgap of $1.12 \mathrm{eV}$. Although indium gallium arsenide (InGaAs) or silicon-germanium ( $\mathrm{SiGe}$ ) photodetectors can be used instead of $\mathrm{Si}$, it is still difficult to fabricate high-quality substrates of large size without defects, and the cost of these materials is much higher than that of Si. Consequently, applications using NIR light are limited in some specific situations despite its potentially excellent ability. Therefore, Si-based plasmonic Schottky devices have attracted a great deal of interest to realize useful NIR detectors $3,4,8,11,12,14-17$.

Until now, a major focus in the development of plasmonic Schottky devices has been to improve the efficiency of light absorption by fabricating properly designed metallic nanostructures $8,11,12,16$. In lithography, a Ti adhesion layer has often been used to enhance the bonding between the metal film and Si substrate; however, the Ti adhesion layer decreases the plasmonic properties and the efficiency of hot 
electron injection due to an increase in electron-phonon coupling and acceleration in the electronphonon relaxation rate ${ }^{18-20}$. In this study, we attempt to improve the responsivities of versatile Si-based photodetectors in the NIR region based on three strategies: (i) alloying plasmonic materials to control the Schottky barrier height, (ii) fabricating an alloy layer on Si to increase the area of the Schottky interface for electron transfer, and (iii) fabricating plasmonic alloy NPs with high coverage to increase light absorption. Using these strategies, we successfully achieve high internal quantum efficiencies (IQEs) of $3.3 \%(0.52 \%)$ and responsivities of $7.3 \mathrm{~mA} / \mathrm{W}(1.9 \mathrm{~mA} / \mathrm{W})$ at a telecommunication wavelength of 1310 $\mathrm{nm}(1550 \mathrm{~nm})$ without external bias. The IQE is $4.6(6.5)$ times higher than those in previous reports, and these responsivities are a record high. These results indicate that the hot electron injection efficiency is notably enhanced owing to the controlled Schottky barrier height and large area of the Schottky interface for electron transfer.

\section{Results}

\section{Device fabrication and nanostructural analysis}

In plasmonic Schottky devices, the Schottky barrier has extremely important roles in separating hot electrons and holes and generating a rectified photocurrent; notably, the injected hot electrons are accelerated by an electric field in the depletion region of Si. However, an excessively high Schottky barrier decreases the efficiency of hot electron injection, as shown in Fig. 1a. Neglecting the metalsemiconductor interaction, the Schottky barrier height is determined by the difference between the work function of the metal and the electron affinity of the semiconductor. In the case of Au, which is the most commonly used plasmonic material because of its efficient plasmon absorption and stability, combined with $\mathrm{n}$-type $\mathrm{Si}$, the Schottky barrier height is estimated to be approximately $0.7 \mathrm{eV}^{21}$. This value is excessively high, especially when electrons are excited by NIR light with a photon energy below $\sim 1.0 \mathrm{eV}$. This is a reason for the low responsivities in the NIR region even in the previously reported Si-based plasmonic Schottky devices, which show highly efficient light absorption $(>90 \%)$. One of the ways to control the energy band diagram in devices is to induce an external bias voltage; however, it causes high noise due to an increased dark current. Therefore, it is extremely important to control the Schottky barrier height without an external bias voltage (Fig. 1b). We control the Schottky barrier height by adjusting the work function of plasmonic nanoalloys by combining them with n-type Si substrates because alloying allows control of the electronic structure 22,23 . We adopt $\mathrm{Ag}$ as a solute element to Au because Ag has a smaller work function than $\mathrm{Au}^{21}$ and is also an excellent plasmonic material. However, $\mathrm{Si}$ is a well-known semiconductor that strongly receives the effect of Fermi-level pinning owing to its small ionicity ${ }^{24-26}$. This means that it is difficult to control the Schottky barrier height even if the work functions of the metals combined with Si are changed. In recent studies, it has been reported that the Schottky barrier height can be controlled by suppressing Fermi-level pinning by direct connection between the metal and semiconductor with atomically flat and extremely clean surfaces, leading to a decrease in the density of the interface states even in the case of using semiconductors with small ionicity values that are similar to $\mathrm{Si}^{27-29}$. In this report, we directly fabricate AuAg nanoalloys on the surface of Si substrates by cathodic 
arc plasma deposition for the first time (Fig. 1C). This simple fabrication method achieves advantageous low-cost and large-scale manufacturing. In addition, it is worth noting that the plasmonic nanostructures can be fabricated in only $\sim 1 \mathrm{~min}$.

Previous works reported Si-based plasmonic Schottky devices with properly designed nanoantennas fabricated by nanofabrication processing using electron-beam lithography 8,11,12,15-17 or Au NPs fabricated by a thermal dewetting process of Au thin film on Si substrates ${ }^{3,4}$. In most of them, $\mathrm{Si}(100)$ substrates were conventionally used, and the surface was not been carefully treated; $\mathrm{HF}$ treatment to remove native oxides increases the microroughness and density of surface states. On the other hand, we prepared an atomically flat and extremely clean Si(111) surface (Supplementary Fig. 1 ) through simple $\mathrm{NH}_{4} \mathrm{~F}$ treatment, including the removal process of a native oxide laye ${ }^{30,31}$. The hydrogen-terminated atomically flat $\mathrm{Si}(111)$ surface could passivate the surface against oxidation. After the cleaning process of $\mathrm{Si}(111)$ substrates, we directly fabricated AuAg nanoalloys on the surface by cathodic arc plasma deposition, which was recently studied to fabricate metal NP catalysts on supports or substrates ${ }^{32-36}$. Fig. 2 shows cross-sectional transmission electron microscopy (TEM) images of the devices fabricated with different numbers of arc plasma pulses. With 10 pulses (Fig. 2a), two layers were formed on the surface of the Si substrate. The upper layer was found to be amorphous. After more than 20 pulses (Fig. $2 b-d$ ), NPs were formed on the amorphous layer. With 40 pulses (Fig. 2c, Supplementary Figs. 2 and 3), many NPs were formed with high coverage, and with 80 pulses (Fig. 2d), the NPs were connected. Thus, we succeeded in controlling the coverage of NPs on the Si substrates. Such nanostructures can be uniformly observed at the interfaces in a larger area (Supplementary Fig. 2). The condition of the cathodic arc plasma deposition was optimized with 40 pulses because the responsivity of the fabricated device was the highest at photocurrent measurements (Supplementary Figs. 4 and 5).

To investigate the detailed morphology, scanning TEM (STEM) analysis was carried out. Fig. 3a shows a cross-sectional high-angle annular dark-field STEM (HAADF-STEM) image of the device fabricated with 40 arc plasma pulses. Fig. 3b-f show the STEM energy-dispersive X-ray spectroscopy (EDX) maps corresponding to Au- $L, \mathrm{Ag}-L, \mathrm{Si}-K, \mathrm{O}-K$, and In- $L$. These results indicated that the layer on the surface of the Si substrate consists of AuAg alloy. From the EDX data shown in Supplementary Fig. 6, the composition of the alloy layer was $\mathrm{Au}: \mathrm{Ag}=0.84: 0.16$, while that of the target of the cathodic arc plasma deposition was $\mathrm{Au}: \mathrm{Ag}=0.80: 0.20$. In addition, the amorphous layer on the AuAg layer consisted of $\mathrm{SiO}_{2}$ (Supplementary Fig. 7), and the NPs consisted of the AuAg alloy. The composition of the alloy NPs was $\mathrm{Au}: \mathrm{Ag}=0.86: 0.14$ (Supplementary Fig. 6). The composition of the AuAg nanoalloy was controlled by the target of cathodic arc plasma deposition (Supplementary Table 1, Supplementary Fig. 8). Moreover, in this deposition method, positively charged metal atoms were released from the target, and the coverage of the deposited NPs could be controlled by the number of pulses ${ }^{35,36}$. The formation of isolated metallic NPs with high coverage was effective in increasing light absorption by plasmon resonance. The thicknesses of the AuAg and $\mathrm{SiO}_{2}$ layers were approximately $2.1 \mathrm{~nm}$ and $2.4 \mathrm{~nm}$, respectively, and the size of the NPs was approximately $4.0 \mathrm{~nm}$. The length of the nanostructure on the surface of $\mathrm{Si}$ is sufficiently short compared with the mean free path $(\sim 50 \mathrm{~nm})$ of hot electrons in $\mathrm{Au}$ and/or $\mathrm{Ag}^{37}$. In 
addition, STEM-electron energy-loss spectroscopy (STEM-EELS) was carried out to investigate more detailed morphology at high space and energy resolution. As shown in Supplementary Fig. 9, the shift in the peaks of the Si- $L_{2,3}$ edge between the area of 3-6 and 8-12 was observed, and the peak was not observed at the area of 7. In addition, the EELS spectra at the Si- $L_{2,3}$ edge could be classified into two kinds of components, which were derived from Si semiconductors and $\mathrm{SiO}_{2}$ (Supplementary Fig. 10a). Supplementary Fig. 10c and d show the cross-sectional STEM-EELS maps of the device. It was confirmed that the AuAg layer hardly contains $\mathrm{Si}$ atoms and that $\mathrm{SiO}_{2}$ was not formed at the interface between the AuAg layer and the Si substrate. These results clearly proved that the AuAg layer directly connected with the atomically flat surface of the Si substrate without appreciable disorder at the interface. The migration of atoms between Au and Si when Au atoms were deposited onto the Si surface has been reported ${ }^{38}$. The structure (AuAg NPs $/ \mathrm{SiO}_{2}$ layer/AuAg layer/Si) might be formed by a similar process due to the reaction at the interface. It is supposed that when a Si amorphous layer consisting of Si atoms migrating from the interface to the surface on the AuAg layer was grown to $2 \mathrm{~nm}$ thickness, the penetration of deposited atoms almost stopped, and NPs were formed on it. The amorphous layer might be oxidized when the sample was exposed to air. Furthermore, electron holography using TEM was performed to investigate the energy band diagram at the interface of Si-based plasmonic Schottky devices for the first time. Fig. $3 \mathrm{~g}$ and $\mathrm{h}$ show the phase image and phase profile across the $\mathrm{Au}_{40} \mathrm{Ag}_{60} / \mathrm{Si}$ interface in the fabricated device without external bias. The phase is directly proportional to the electric potential where the sample thickness was flat, and therefore, these results clearly show that the Schottky barrier is uniformly formed along the whole $\mathrm{Au}_{40} \mathrm{Ag}_{60}$ layer/Si interface. This indicates that hot electrons can be efficiently injected into Si over the Schottky barrier and accelerated by the electric field in the depletion region in the fabricated devices.

\section{Optical characteristic of the fabricated devices}

The optical properties of the fabricated devices were investigated by a spectrophotometer with an integrating sphere. Fig. 4a shows optical absorption spectra of $A u, A u_{80} A_{20}, A u_{60} A g_{40}, A u_{50} A g_{50}$, $\mathrm{Au}_{40} \mathrm{Ag}_{60}, \mathrm{Au}_{20} \mathrm{Ag}_{80}$, and $\mathrm{Ag}$ nanostructures in devices fabricated under the same conditions. The broad light absorption in the NIR region observed in $\mathrm{Au}-\mathrm{Si}$ and $\mathrm{AuAg}-\mathrm{Si}$ devices was attributed to the interaction between plasmonic NPs and Si substrate ${ }^{3,4}$. The absorption spectra do not show a strong dependence on the composition of AuAg nanoalloys. On the other hand, the absorbance in the Ag device was low. This might be because Ag is easily oxidized. This means that the excellent properties of plasmon resonance could be maintained by mixing Au with Ag.

To verify the validity of our concept, photocurrent measurements at telecommunication wavelengths were performed on the fabricated devices without external bias. Fig. $4 \mathrm{~b}$ shows the responsivities at wavelengths of $1310 \mathrm{~nm}$ and $1550 \mathrm{~nm}$ of the $\mathrm{Au}, \mathrm{Au}_{80} \mathrm{Ag}_{20}, \mathrm{Au}_{60} \mathrm{Ag}_{40}, \mathrm{Au}_{50} \mathrm{Ag}_{50}, \mathrm{Au}_{40} \mathrm{Ag}_{60}, \mathrm{Au}_{20} \mathrm{Ag}_{80}$, and $\mathrm{Ag}$ devices. When the composition of Ag was increased from $0 \%$ to $60 \%$, the responsivity was increased, 
and then, the maximum responsivity was $7.3 \mathrm{~mA} / \mathrm{W}$ at $1310 \mathrm{~nm}$ and $1.9 \mathrm{~mA} / \mathrm{W}$ at $1550 \mathrm{~nm}$ in the $\mathrm{Au}_{40} \mathrm{Ag}_{60}$ device. The optical absorption spectra were similar, as shown in Fig. 4a, and therefore, these results indicate that the efficiency of hot electron injection was improved because the Schottky barrier was decreased by alloying Au with Ag, which has a lower work function. When the composition of Ag was further increased to above $80 \%$, the responsivity was decreased. This reason should be that the efficiency

of charge separation was decreased due to the excessively low Schottky barrier, and efficient current flow in one direction was not allowed because injected hot electrons cannot be accelerated due to the weak electric field in the depletion region in Si (Supplementary Fig. 11). In the Ag device, the photocurrent could not be observed due to the decrease in the efficiency of charge separation and low light absorption. To estimate the Schottky barrier height, the quantum transmission probabilities were investigated by the modified Fowler equation as described below ${ }^{39}$,

$$
\eta_{i} \approx C_{\mathrm{F}} \frac{\left(h v-\phi_{\mathrm{b}}\right)^{2}}{h v}
$$

where $C_{\mathrm{F}}$ is the Fowler emission coefficient, is the photon energy, and is the Schottky barrier height. This equation describes the number of hot electrons that can be injected into $\mathrm{Si}$, and it contributes to the photocurrent. Assuming that the responsivities in our devices (Au-Si and AuAg-Si devices) depend only on the quantum transmission probabilities, we compared the results of the experimentally measured responsivities with the calculated quantum transmission probabilities (Fig. 4c) at each wavelength because of similar light absorption. In good agreement between the experimental results of the photocurrent measurements (Fig. 4b) and the calculated results at wavelengths of $1310 \mathrm{~nm}$ and 1550 $\mathrm{nm}$, the Schottky barrier heights at the $\mathrm{Au}-\mathrm{Si}$ and $\mathrm{Au}_{40} \mathrm{Ag}_{60}-\mathrm{Si}$ interfaces were estimated to be $0.70 \mathrm{eV}$ and $0.53 \mathrm{eV}$, respectively. In addition, the IQE of hot electron injection was calculated. Fig. $4 \mathrm{~d}$ shows the IQE values of the Si-based plasmonic Schottky devices in this study and in previous works at wavelengths of $1310 \mathrm{~nm}$ and $1550 \mathrm{~nm}$. The IQE of our device was 3.3\% (0.52\%) at a wavelength of 1310 $\mathrm{nm}(1550 \mathrm{~nm})$, which was 4.6 (6.5) times higher than those of previously reported devices. These results proved that the efficiency of hot electron injection was enhanced by controlling the Schottky barrier. As a result, the responsivity in the NIR region was much improved in comparison with previous works, including those that reported highly efficient light absorbance (>90\%) (see details in Supplementary Table 2 and summary Table 1 in ref. 40).

\section{Discussion}

We showed that the responsivities and IQE values in the NIR region can be enhanced by using AuAg nanoalloys in plasmonic Schottky devices. This can be achieved by controlling the Schottky barrier height owing to the adjustment of the work function and the direct connection at the large Schottky interface between the AuAg nanoalloy layer and Si. In previously reported devices, the Ti adhesion layer with a low work function not only enhances the bonding between the metal film and Si substrate but also decreases the Schottky barrier height $(\sim 0.54 \mathrm{eV})^{16}$, although it strongly receives the effect of Fermi level 
pinning. However, the Ti adhesion layer decreases the plasmonic properties and the efficiency of hot electron injection due to an increase in electron-phonon coupling and acceleration in the electronphonon relaxation rate because $\mathrm{Ti}$ atoms are four times lighter than $\mathrm{Au}$ atoms ${ }^{18-20}$. In contrast, AuAg nanoalloys can realize excellent plasmonic properties and high efficiency of hot electron injection. This is a key to realizing higher responsivity and IQE in comparison with previous reports.

In conclusion, we demonstrated Si-based plasmonic Schottky devices with enhanced hot electron injection facilely fabricated by cathodic arc plasma deposition. In the fabricated AuAg NPs-film structure, the Schottky barrier height was properly controlled by adjusting the composition of the AuAg nanoalloys, and the area of the Schottky interface for electron transfer was increased. As a result, we successfully enhanced the responsivity and IQE of photodetection in the NIR region below the bandgap of Si without external bias. The responsivity can be further enhanced by combining Si nano- and microstructures such as pyramids or trench structures, which are designed to increase light absorption. Our concept in this study can be applied not only to practical photodetectors in the NIR region but also to the use of broader wavelength regions for applications in a variety of fields, such as imaging, photovoltaics and photochemistry.

\section{Methods}

\section{Device fabrication}

The devices were fabricated on lightly doped n-type Si substrates (1-10 $\Omega \mathrm{cm},<111>$ orientation). The Si substrates were cleaned in acetone, and afterwards, organic contamination was removed in a mixed solution of sulfuric acid and hydrogen peroxide $\left(\mathrm{H}_{2} \mathrm{SO}_{4}: \mathrm{H}_{2} \mathrm{O}_{2}=3: 1\right)$. The $\mathrm{Si}$ substrates were immersed in a $40 \% \mathrm{NH}_{4} \mathrm{~F}$ solution without oxygen by adding ammonium sulfite to remove the native oxide and create an atomically smooth hydrogen-terminated surface. Au, Ag, or AuAg nanoalloys were deposited on the front-side surface of the Si substrates by cathodic arc plasma deposition method (Advance RIKO, APD$2 \mathrm{P})$. The arc voltage was $100 \mathrm{~V}$, the condenser capacity was $1080 \mu \mathrm{F}$, and the repetition rate was 1 $\mathrm{Hz}$. The number of arc plasma pulses was optimized by measuring the optical absorption and responsivities (Supplementary Figs. 4 and 5). After that, a tin-doped indium oxide (ITO) film with a thickness of $220 \mathrm{~nm}$ was deposited on the front-side surface of the Si substrates by sputtering. A Ag film with a thickness of $10 \mathrm{~nm}$ was deposited on the back-side surface of the Si substrates as an ohmic contact, and an Al film with a thickness of $50 \mathrm{~nm}$ was deposited on the Ag film for protection.

\section{Characterization}

The cross-sectional TEM and STEM analyses were carried out using a JEOL JEM-ARM200F STEM instrument operated at $200 \mathrm{kV}$. Electron holography was carried out using a $300 \mathrm{kV}$ transmission electron microscope (Hitachi High-Technologies, HF-3300EH) equipped with multiple biprisms. The phase distribution of the electron wave was reconstructed using hologram shifting method ${ }^{41}$ where the phase 
was reconstructed from one hologram using phase-shifting algorithms ${ }^{42}$. For electron holography, the devices were fabricated on lightly doped $n$-type Si substrates $(0.08-0.1 \Omega \mathrm{cm},<111>$ orientation). Therefore, the depletion layer should be slightly short compared with those of devices investigated in other measurements. The samples were fabricated by focused ion beam (FIB) milling. Atomic force microscopy (AFM) images were obtained using a Pacific Nanotechnology Nano-RJ. X-ray photoelectron spectroscopy (XPS) analyses were carried out using a ULVAC PHI Quantera-SXM. Absorption spectra were obtained using a UV-VIS-NIR spectrophotometer (Shimadzu, SolidSpec-3700DUV) with an integrating sphere. The measurements of the photocurrent were performed using an electrochemical analyser (ALS, Model 660E). The laser at wavelengths of $1310 \mathrm{~nm}$ or $1550 \mathrm{~nm}$ with a spot size of $\sim 900 \mu \mathrm{m}$ was irradiated to the front-side surface of the device at room temperature.

IQE calculation. The IQE percentage was calculated by Equation (2),

$$
I Q E=100 \times \frac{I_{\mathrm{p}} / q}{P_{\mathrm{ab} / h v}}
$$

where $I_{\mathrm{p}}$ is the photocurrent, $q$ is the electron charge, $P_{\mathrm{ab}}$ is the power of absorbed light and $h v$ is the photon energy ${ }^{12}$.

\section{Declarations}

\section{Author contributions}

S.O., K.K., T.H. and H.K. conceived and designed the research. S.O. fabricated the devices and characterized their morphology and properties. Y.N., S.A. and K.Y. performed the TEM holography analyses. E.T. performed the XPS analyses. All the authors discussed the experimental results and contributed to the preparation of the manuscript.

Correspondence and requests for materials should be addressed to S.O.

(okamoto.shinya001@jp.panasonic.com)

\section{Competing interests}

The authors declare no competing financial interests.

\section{References}


1. Clavero, C. Plasmon-induced hot-electron generation at nanoparticle/metal-oxide interfaces for photovoltaic and photocatalytic devices. Nat. Photonics 8, 95-103 (2014).

2. Brongersma, M. L., Halas, N. J. \& Nordlander, P. Plasmon-induced hot carrier science and technology. Nat. Nanotechnol. 10, 25-34 (2015).

3. Nazirzadeh, M. A., Atar, F. B., Turgut, B. B. \& Okyay, A. K. Random sized plasmonic nanoantennas on silicon for low-cost broad-band near-infrared photodetection. Sci. Rep. 4, 7103 (2014).

4. Qi, Z. et al. Au nanoparticle-decorated silicon pyramids for plasmon-enhanced hot electron nearinfrared photodetection. Nanotechnology 28, 275202 (2017).

5. Nakamura, K. et al. Properties of plasmon-induced photoelectric conversion on a TiO2/NiO p-n junction with Au nanoparticles. J. Phys. Chem. Lett. 7, 1004-1009 (2016).

6. Zhong, Y., Ueno, K., Mori, Y., Oshikiri, T. \& Misawa, H. Cocatalyst effects on hydrogen evolution in a plasmon-induced water-splitting system. J. Phys. Chem. C119, 8889-8897 (2015).

7. Ding, D., Liu, K., He, S., Gao, C. \& Yin, Y. Ligand-exchange assisted formation of Au/TiO2 Schottky contact for visible-light photocatalysis. Nano Lett. 14, 6731-6736 (2014).

8. Knight, M. W., Sobhani, H., Nordlander, P. \& Halas, N. J. Photodetection with active optical antennas. Science 332, 702-704 (2011).

9. Ueno, K. \& Misawa, H. Plasmon-enhanced photocurrent generation and water oxidation from visible to near-infrared wavelengths. NPG Asia Mater. 5, e61 (2013).

10. Nishijima, Y. et al. Near-infrared plasmon-assisted water oxidation. J. Phys. Chem. Lett. 3, 12481252 (2012).

11. Li, W. et al. Circularly polarized light detection with hot electrons in chiral plasmonic metamaterials. Nat. Commun. 6, 8379 (2015).

12. Sobhani, A. et al. Narrowband photodetection in the near-infrared with a plasmon-induced hot electron device. Nat. Commun. 4, 1643 (2013).

13. Giugni, A. et al. Hot-electron nanoscopy using adiabatic compression of surface plasmons. Nat. Nanotechnol. 8, 845-852 (2013).

14. Wen, L., Chen, Y., Liang, L. \& Chen, Q. Hot electron harvesting via photoelectric ejection and photothermal heat relaxation in hotspots-enriched plasmonic/photonic disordered nanocomposites. ACS Photonics 5, 581-591 (2018).

15. Lin, K. T., Chen, H. L., Lai, Y. S. \& Yu, C. C. Silicon-based broadband antenna for high responsivity and polarization-insensitive photodetection at telecommunication wavelengths. Nat. Commun. 5, 3288 
(2014).

16. Li, W. \& Valentine, J. Metamaterial perfect absorber based hot electron photodetection. Nano Lett. 14, 3510-3514 (2014).

17. Lin, K. T. et al. Silicon-based embedded trenches of active antennas for high-responsivity omnidirectional photodetection at telecommunication wavelengths. ACS Appl. Mater. Interfaces 11, 31503159 (2019).

18. Habteyes, T. G. et al. Metallic adhesion layer induced plasmon damping and molecular linker as a nondamping alternative. ACS Nano 6, 5702-5709 (2012).

19. Giri, A. et al. Mechanisms of nonequilibrium electron-phonon coupling and thermal conductance at interfaces. J. Appl. Phys. 117, 105105 (2015).

20. Zhou, X. et al. Strong influence of Ti adhesion layer on electron-phonon relaxation in thin gold films: Ab initio nonadiabatic molecular dynamics. ACS Appl. Mater. Interfaces 9, 43343-43351 (2017).

21. Sze, S. M. \& Ng, Kwok K. Metal-semiconductor contacts in Physics of Semiconductor Devices 134196 (John Wiley \& Sons, Inc, 2007).

22. Kusada, K. \& Kitagawa, H. A route for phase control in metal nanoparticles: a potential strategy to create advanced materials. Adv. Mater. 28, 1129-1142 (2016).

23. Kusada, K. et al. Solid solution alloy nanoparticles of immiscible Pd and Ru elements neighboring on Rh: changeover of the thermodynamic behavior for hydrogen storage and enhanced CO-oxidizing ability. J. Am. Chem. Soc. 136, 1864-1871 (2014).

24. Kurtin, S., McGill, T. C. \& Mead, C. A. Fundamental transition in the electronic nature of solids. Phys. Rev. Lett. 22, 1433-1436 (1969).

25. Tersoff, J. Schottky barrier heights and the continuum of gap states. Phys. Rev. Lett. 52, 465-468 (1984).

26. Tung, R. T. The physics and chemistry of the Schottky barrier height. Appl. Phys. Rev. 1, 011304 (2014).

27. Zhou, Y. et al. Investigating the origin of Fermi level pinning in Ge Schottky junctions using epitaxially grown ultrathin MgO films. Appl. Phys. Lett. 96, 102103 (2010).

28. Yang, H. et al. Graphene barristor, a triode device with a gate-controlled Schottky barrier. Science 336, 1140-1143 (2012).

29. Teraji, T. \& Hara, S. Control of interface states at metal/6H-SiC (0001) interfaces. Phys. Rev. B 70, 035312 (2004). 
30. Kato, H. et al. Preparation of an ultraclean and atomically controlled hydrogen-terminated $\mathrm{Si}(111)-$ $(1 \times 1)$ surface revealed by high resolution electron energy loss spectroscopy, atomic force microscopy, and scanning tunneling microscopy: aqueous NH4F etching process of Si (111). Jpn. J. Appl. Phys. 46, 5701-5705 (2007).

31. Angermann, H. et al. Wet-chemical passivation of atomically flat and structured silicon substrates for solar cell application. Appl. Surf. Sci. 254, 3615-3625 (2008).

32. Kim, S. H. et al. Catalytic activity of $\mathrm{Au} / \mathrm{TiO} 2$ and $\mathrm{Pt} / \mathrm{TiO} 2$ nanocatalysts prepared with arc plasma deposition under CO oxidation. Appl. Catal. A Gen. 454, 53-58 (2013).

33. Fujitani, T., Nakamura, I., Akita, T., Okumura, M. \& Haruta, M. Hydrogen dissociation by gold clusters. Angew. Chem. Int. Ed. Engl. 48, 9515-9518 (2009).

34. Qadir, K., Kim, S. H., Kim, S. M., Ha, H. \& Park, J. Y. Support effect of arc plasma deposited Pt Nanoparticles/TiO2 substrate on catalytic activity of CO oxidation. J. Phys. Chem. C116, 24054-24059 (2012).

35. Kim, S. H., Moon, S. Y. \& Park, J. Y. Non-colloidal nanocatalysts fabricated using Arc plasma deposition and their application in heterogenous catalysis and photocatalysis. Top. Catal. 60, 812-822 (2017).

36. Kim, S. H., Jeong, Y. E., Ha, H., Byun, J. Y. \& Kim, Y. D. Ultra-small platinum and gold nanoparticles by Arc plasma deposition. Appl. Surf. Sci. 297, 52-58 (2014).

37. Brown, A. M., Sundararaman, R., Narang, P., Goddard, W. A., 3rd \& Atwater, H. A. Nonradiative plasmon decay and hot carrier dynamics: effects of phonons, surfaces, and geometry. ACS Nano 10, 957966 (2016).

38. Yeh, J. et al. Growth of the room temperature Au/Si(111)-7 x 7 interface. Phys. Rev. Lett. 70, 37683771 (1993).

39. Fowler, R. H. The analysis of photoelectric sensitivity curves for clean metals at various temperatures. Phys. Rev. 38, 45-56 (1931).

40. Lin, K. T., Lin. H. \& Jia, B. Plasmonic nanostructures in photodetection, energy conversion and beyond. Nanophotonics 9, 3135-3163 (2020).

41. Ru, Q., Hirayama, T., Endo, J. \& Tonomura, A. Hologram-shifting method for high-speed electron hologram reconstruction. Jpn. J. Appl. Phys. 31, 1919-1921 (1992).

42. Yamamoto, K., Kawajiri, I., Tanji, T., Hibino, M. \& Hirayama, T. High precision phase-shifting electron holography. J. Electron Microsc. 49, 31-39 (2000). 
a

b

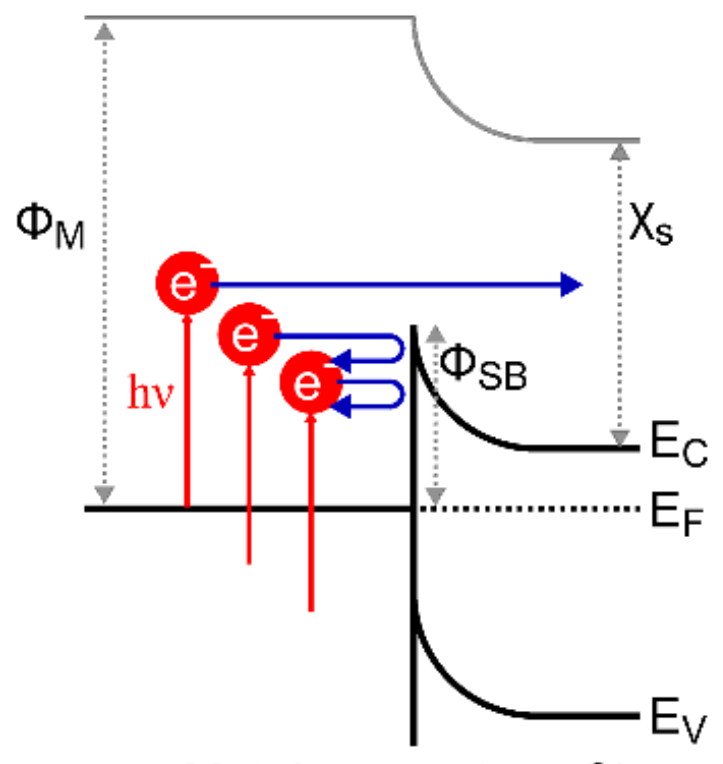

Metal

n-type Si

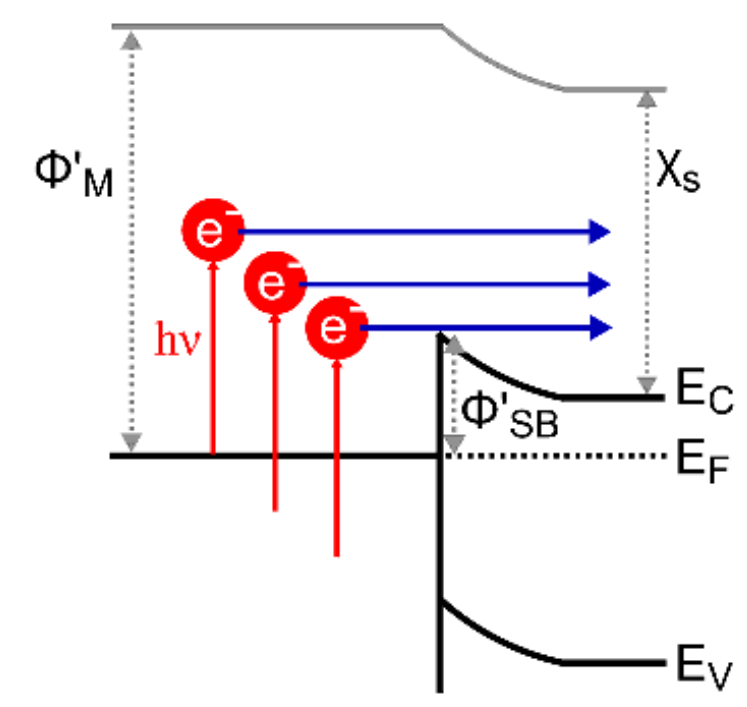

Metal

n-type Si

C

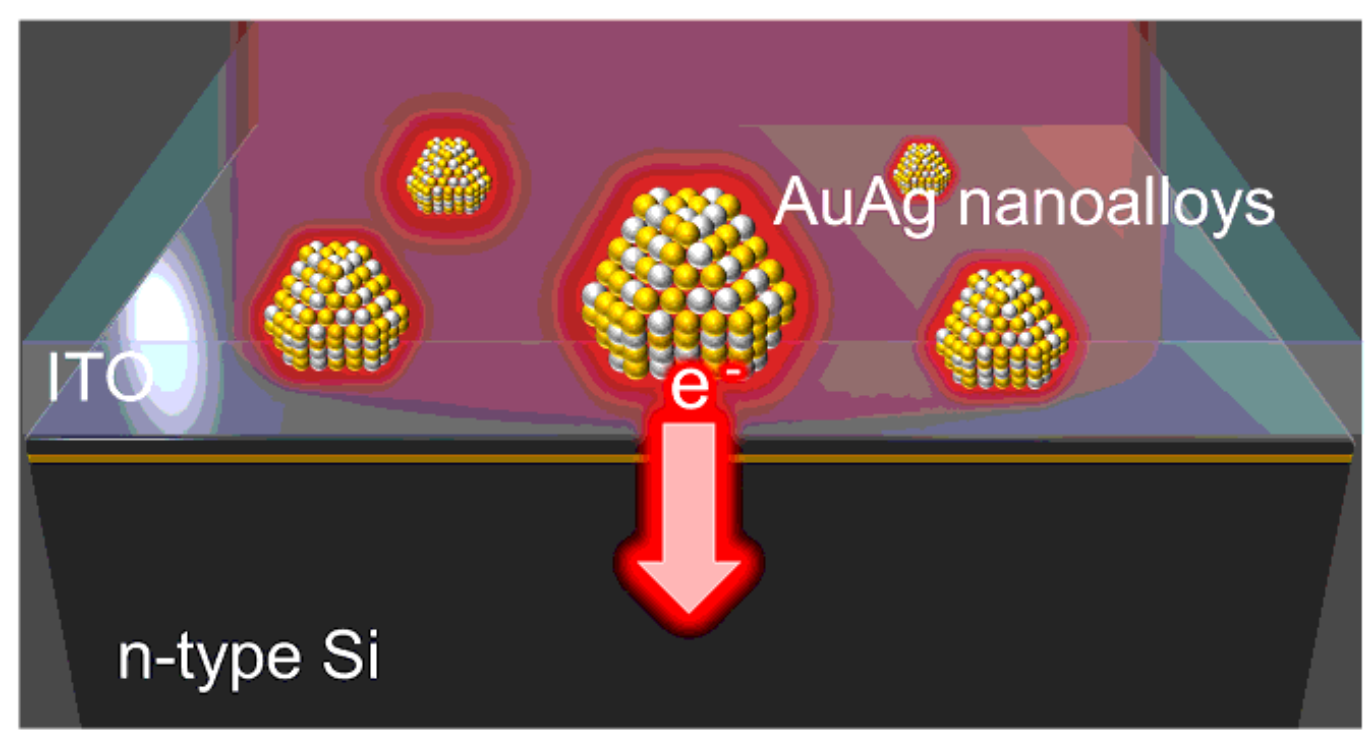

Figure 1

Plasmon-induced hot electron injection in a plasmonic Schottky device. Energy band diagram of a plasmonic photodetector with an (a) excessively high and a (b) properly decreased Schottky barrier $\left(\Phi_{\mathrm{SB}}\right.$, 
$\left.\Phi_{S B}^{\prime}\right)$, where $\Phi_{M}$ and $\Phi_{M}^{\prime}$ are the work functions of the metals, $\chi_{S}$ is the electron affinity of the semiconductor, $E_{F}$ is the Fermi level, $E_{V}$ is the valence band and $E_{c}$ is the conduction band of the semiconductor. In the case of n-type Si $(1-10 \Omega \mathrm{cm}), \mathrm{E}_{\mathrm{c}}-\mathrm{E}_{\mathrm{f}} \approx 0.28 \mathrm{eV}$. (c) Schematic of the fabricated AuAgSi plasmonic Schottky device.

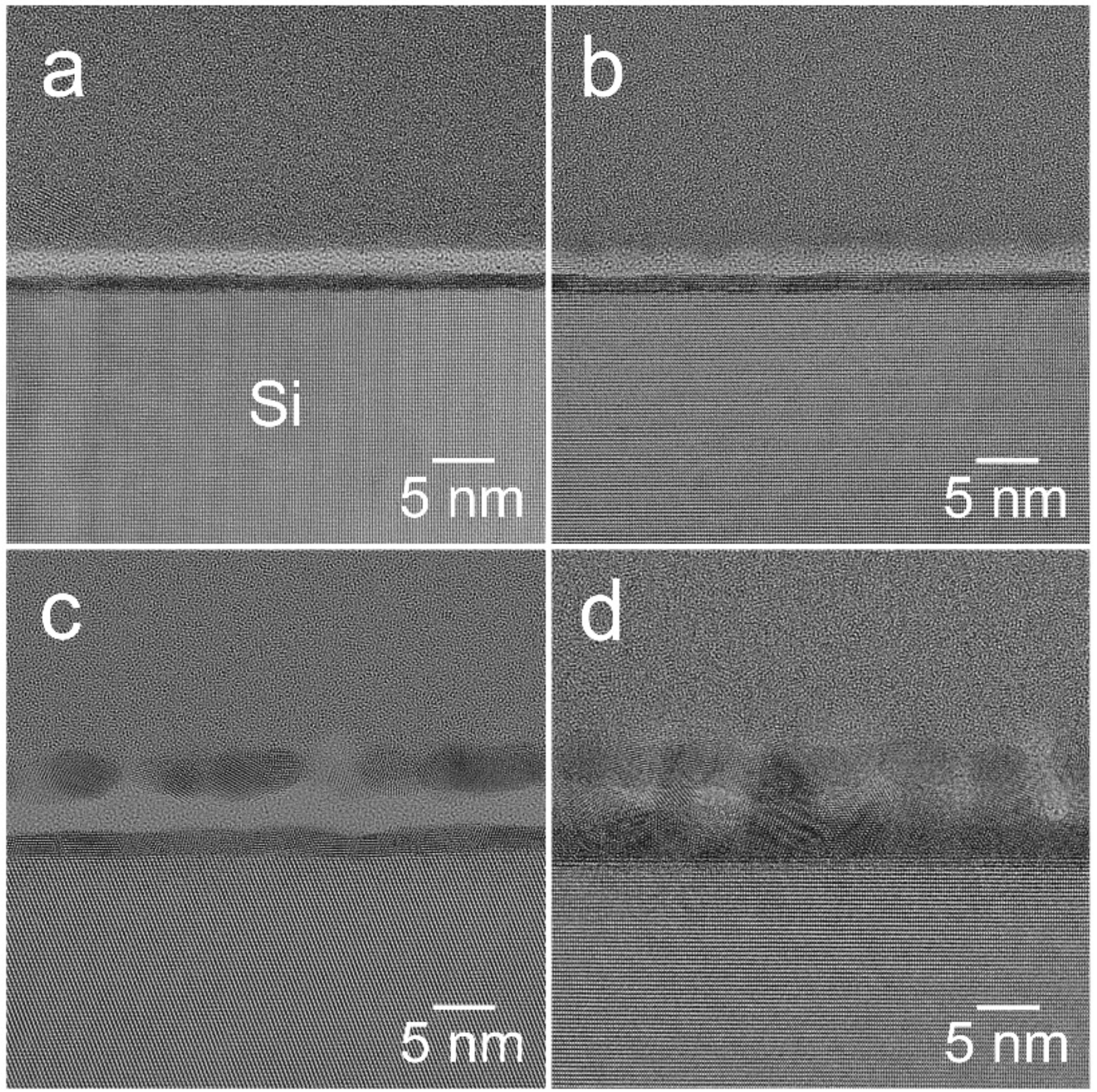

Figure 2 
TEM analysis of nanostructures on the Si substrate. Cross-sectional TEM images of the devices fabricated with (a) 10, (b) 20, (c) 40, and (d) 80 arc plasma pulses.
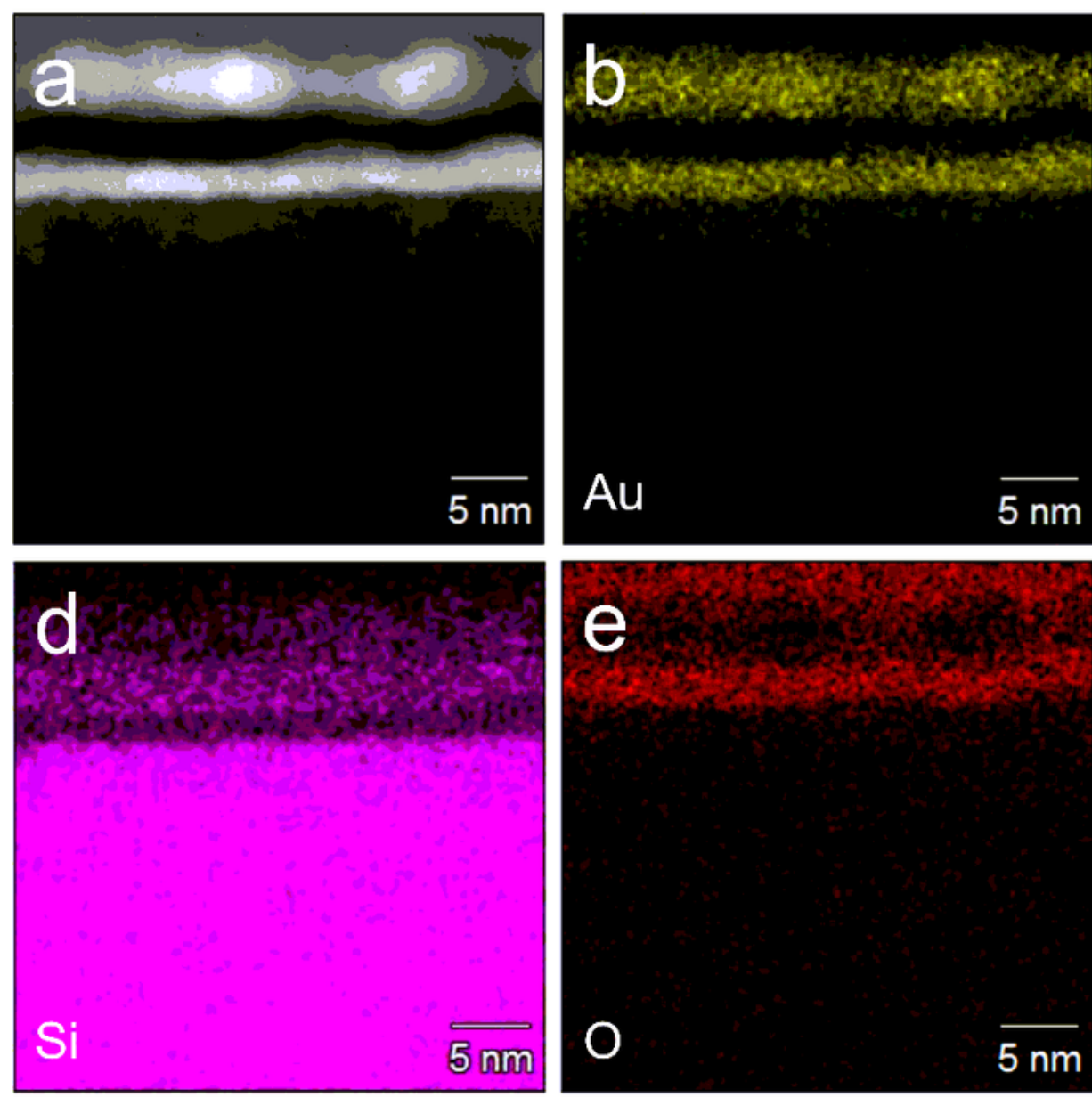

e

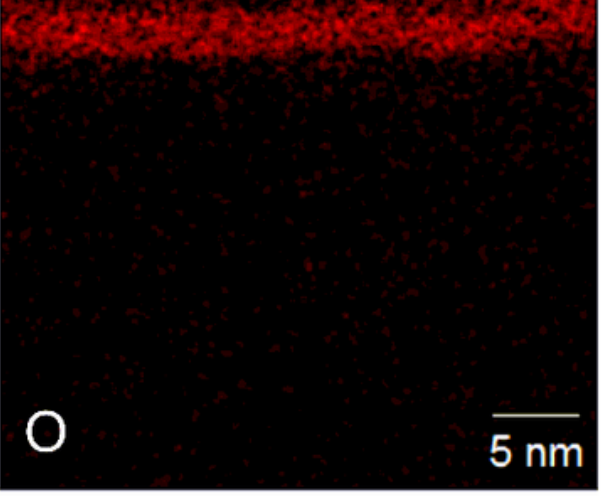

In
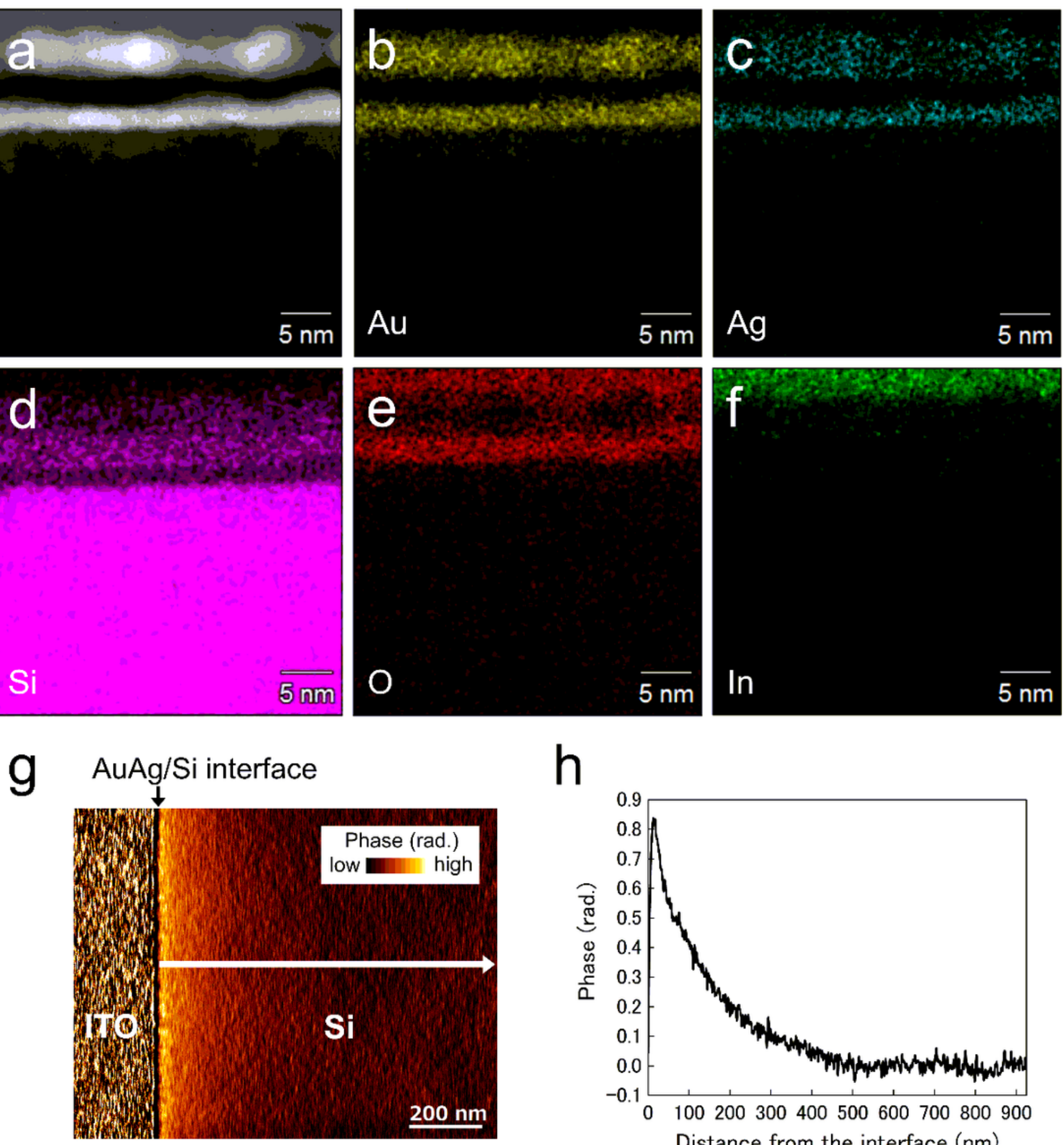

$\mathrm{h}$

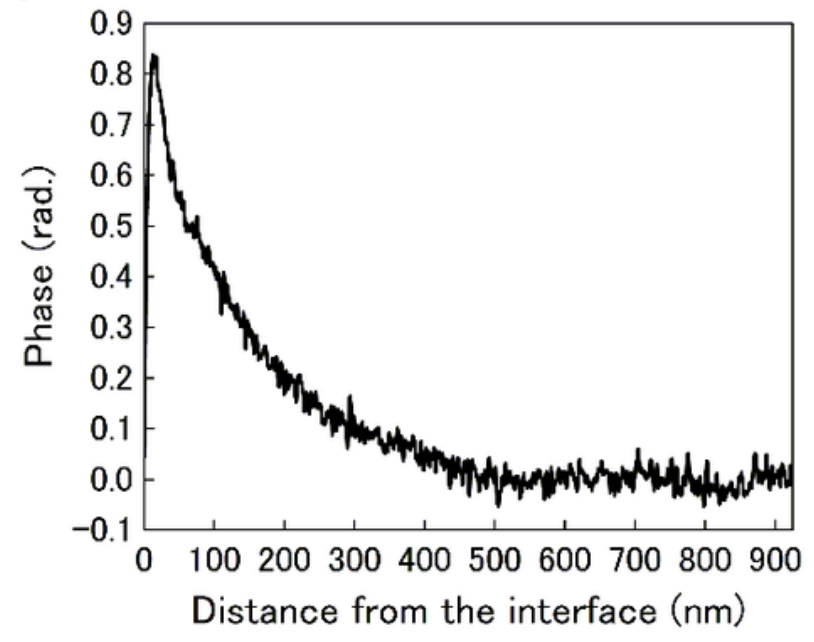

Figure 3

STEM-EDX analysis and electron holography. (a) Cross-sectional HAADF-STEM image. (b) Au- $L$, (c) Ag- $L$, (d) Si-K, (e) O-K and (f) In- $L$ STEM-EDX maps of (a). (g) Phase image. (h) Phase profile along the arrow 
shown in (g).
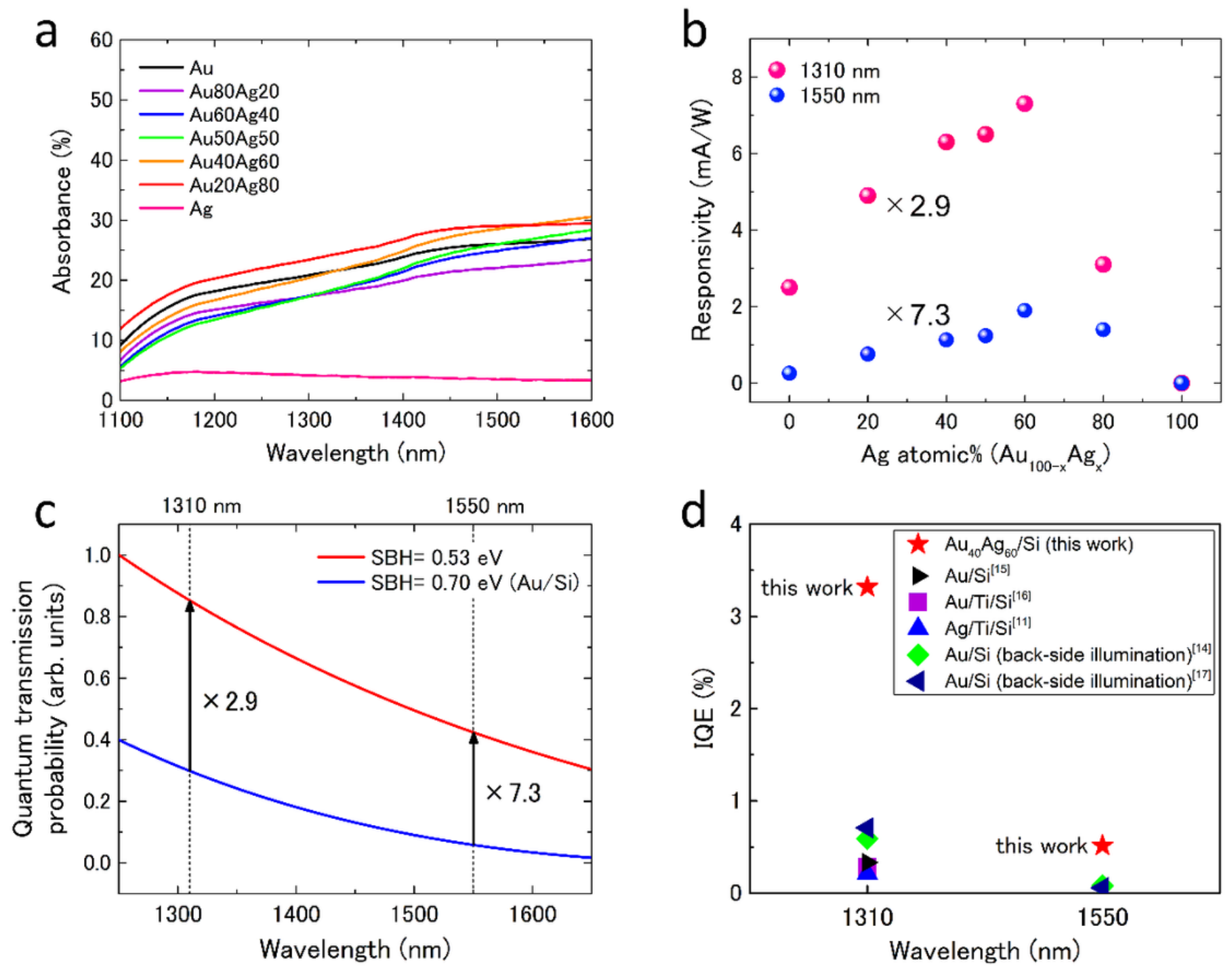

\section{Figure 4}

Device properties (a) Absorption spectra of the metal nanostructures consisting of $A u, A u_{80} A_{9}$, $\mathrm{Au}_{60} \mathrm{Ag}_{40}, \mathrm{Au}_{50} \mathrm{Ag}_{50}, \mathrm{Au}_{40} \mathrm{Ag}_{60}, \mathrm{Au}_{20} \mathrm{Ag}_{80}$, or $\mathrm{Ag}$. (b) Responsivities at wavelengths of $1310 \mathrm{~nm}$ (pink dots) and $1550 \mathrm{~nm}$ (blue dots) of devices fabricated with different compositions. (c) Calculated quantum transmission probabilities of a Schottky barrier height (SBH) of $0.70 \mathrm{eV}$ at the Au/Si interface (blue line) and $0.53 \mathrm{eV}$ (red line). (d) IQE of Si-based plasmonic Schottky devices in this work and previous reports at wavelength of $1310^{11,14-17} \mathrm{~nm}$ and $1550^{14,17} \mathrm{~nm}$, respectively.

\section{Supplementary Files}

This is a list of supplementary files associated with this preprint. Click to download.

- SupplementaryInfoS020220114.docx 